IJMMS 30:12 (2002) 717-725

PII. S0161171202109197

http://ijmms.hindawi.com

(c) Hindawi Publishing Corp.

\title{
CUT POINTS IN ABCOHESIVE, APOSYNDETIC, AND SEMI-LOCALLY CONNECTED SPACES
}

\author{
DAVID A. JOHN and SHING S. SO
}

Received 17 September 2001

\begin{abstract}
In 1941, F. B. Jones introduced aposyndesis, which generalizes the concept of semi-local connectedness defined earlier by G. T. Whyburn (1942), in the study of continuum theory. Using Jones's idea, D. A. John (1993) defined abcohesiveness as a generalization of aposyndesis and studied the $A$-sets in abcohesive spaces. In this paper, some properties of abcohesive spaces are studied and a number of results by B. Lehman (1976) and Whyburn $(1942,1968)$ are generalized; sufficient conditions for the existence of two nodal sets are established as well.
\end{abstract}

2000 Mathematics Subject Classification: 54D05.

\section{Aposyndetic and abcohesive spaces}

DEFINITION 1.1. A space $M$ is aposyndetic at a point $p$ with respect to a point $q$ if and only if there exists a closed connected set $H$ such that $p \in \operatorname{int}(H)$ and $H \subseteq M-\{q\}$. The space $M$ is aposyndetic at a point $p$ if and only if it is aposyndetic at $p$ with respect to $q$ for each $q$ in $M-\{p\}$. The space is aposyndetic if and only if it is aposyndetic at $p$ for each $p$ in $M$.

A space $M$ is abcohesive at a point $p$ with respect to a point $q$ if and only if there exists an open connected set $U$ such that $p \in U$ and $U \subseteq M-\{q\}$. The space $M$ is abcohesive at a point $p$ if and only if it is abcohesive at $p$ with respect to $q$ for each $q$ in $M-\{p\}$. The space is abcohesive if and only if it is abcohesive at $p$ for each $p$ in $M$.

John [2] showed that aposyndesis implies abcohesiveness and hence abcohesivness is also a generalization of semi-local connectedness. Besides the properties discussed in [2], the next three theorems show some additional properties of abcohesive spaces. All spaces in this paper are assumed to be topological.

THEOREM 1.2. (a) Every quasicomponent of an abcohesive space is open and is also a component of the space.

(b) If the sets $H$ and $K$ are abcohesive at $p$, then $H \cup K$ is abcohesive at $p$.

(c) If $M$ is a Hausdorff space, $S$ is an open subset of $M$ such that $S$ is abcohesive, then $\bar{S}$ is abcohesive at every point $p \in S$ with respect to every point $q \in \bar{S}-S$.

Proof. (a) Let $Q$ be a quasicomponent of the abcohesive space $M$. If $M$ is connected, then $M$ is the only component of the space so $Q=M$. If $M$ is disconnected, let $C$ be a component in $M$ such that $C \cap Q \neq \varnothing$. Since $C \neq M$, let $p \in M-C$. Then $C$ is 
a component of $M-\{p\}$ and hence $C$ is open by [2, Theorem 1]. Since $C$ is both open and closed, $Q \subseteq C$. But, $C$ is a subset of a quasicomponent of $M$, therefore $C=Q$.

(b) For each $q \in H \cup K-\{p\}, q \in H-\{p\}$ or $q \in K-\{p\}$ so there exist open and connected sets $U$ and $V$ such that $q \in U \subset H-\{p\}$ or $q \in V \subset K-\{p\}$. Thus $H \cup K$ is abcohesive.

(c) Let $p \in S$ and $q \in \bar{S}-S$. Since $M$ is Hausdroff, there exist disjoint open sets $U_{p}$ and $V_{q}$ such that $p \in U_{p}$ and $q \in V_{q}$. Since $q \in \bar{S}-S$, there exists $q^{*} \in S$ such that $q^{*} \in V_{q}$. Since $S$ is open and abcohesive, there exists an open connected set $U$ of $M$ such that $p \in U, U \subset S-q^{*}$, and $q \notin U$ because $q \in \bar{S}-S$. Therefore, $p \in U$ and $U \subset \bar{S}-\left\{q, q^{*}\right\} \subset \bar{S}-\{q\}$.

The next theorem is a characterization of abcohesive spaces.

THEOREM 1.3. A space is abcohesive if and only if each of its quasicomponents is open and abcohesive.

Proof. Let $Q$ be a quasicomponent of the abcohesive space $M$. By Theorem 1.2(a), it suffices to show that $Q$ is abcohesive. For each $p \in Q$ and $q \in Q-\{p\}$, there exists an open connected set $U$ such that $p \in U$ and $U \subseteq M-\{q\}$ because $M$ is abcohesive. Since $p \in U$ and $U$ is connected, by Theorem 1.2(a) $U \subset Q$ and hence $U$ is an open connected set in $Q-\{q\}$. Therefore, $Q$ is abcohesive.

Conversely, assume that every quasicomponent of $M$ is open and abcohesive. For each $p \in M$ and $q \in M-\{p\}$, let $Q$ be the quasicomponent of $M$ containing $p$ and assume that $M \neq Q$. Let $q \notin Q$. Since $p \in Q, Q \subset M-\{q\}$, and $Q$ is open and connected by Theorem 1.2(a), $M$ is abcohesive at $p$ with respect to $q$. Let $q \in Q$. Since $Q$ is abcohesive there exists an open connected subset $U$ of $Q$ containing $p$ such that $U \subset Q-\{q\} \subset M-\{q\}$. Since $Q$ is open in $M$ and $U$ is open in $Q, U$ is open in $M$. Thus, $U$ is an open connected set in $M$ containing $p$ such that $U \subset M-\{q\}$ so $M$ is abcohesive at $p$ with respect to $q$ for each $q \in Q-\{p\}$ and $p \in Q$. Therefore, $M$ is abcohesive.

Definition 1.4. Let $M$ be a connected space. A point $p$ is a cut point of $M$ if and only if $M-\{p\}$ is disconnected. A point $p$ is an end point of $M$ if and only if each open set containing $p$ contains an open set containing $p$ whose boundary is degenerate. Two points $a, b$ in a space $M$ are conjugate in $M$ if no point of $M$ separate $a$ and $b$.

Definition 1.5. An $A$-set $A$ of a space $M$ is a closed subset of $M$ such that $M-A$ is the union of a collection of open sets each bounded by a single point of $A$.

DEFINITION 1.6. Let $a$ and $b$ be points of a space $M$. Then $C(a, b)$ denotes the intersection of all $A$-sets of $M$, which contain both $a$ and $b$, and the set $C(a, b)$ is called the cyclic chain in $M$ from $a$ to $b$.

THEOREM 1.7. If $a$ and $b$ are two distinct conjugate points of an abcohesive connected space $M$ and $C(a, b) \neq \varnothing$, then $C(a, b)=\{x \in M: x$ is conjugate to both $a$ and $b$.

Proof. Let $x$ be conjugate to both $a$ and $b$ and assume $a \notin A$ for some $A$-set $A$ of $M$ containing $a$ and $b$. Let $K$ be the component of $M-A$ containing $x$. By 
[2, Theorem 12], $K$ is open, $\partial K=\{p\}$ for some $p$ in $A$, and $K$ is a component of $M-\{p\}$. Now, $M-\{p\}=K \cup[(M-\{p\})-K]$ where $K$ and $(M-\{p\})-K$ are separated with $x \in K$ and $\{a, b\} \cap[(M-\{p\})-K] \neq \varnothing$. Thus, $x$ is not conjugate to $a$ or $b$, which is a contradiction.

Let $x \in A$ for all $A$-sets $A$ of $M$ containing $a$ and $b$. Suppose $x$ is not conjugate to $a$. Then there exists $p \in M$ such that $M-\{p\}=M_{x} \cup M_{a}$ where $M_{x}$ and $M_{a}$ separated with $x \in M_{x}$ and $a \in M_{a}$. Let $K^{*}$ be the component of $M-\{p\}$ containing $a$. If $b \neq p$ and $b \in M_{x}$, then it contradicts the fact that $a$ and $b$ are distinct conjugate points of $M$. If $b=p$ or $b \in M_{a}$, then $K^{*} \cup\{p\}$ is an $A$-set of $M$ containing both $a$ and $b$ according to [2, Theorem 14], so $x \in K^{*} \cup\{p\}$, which is a contradiction.

The proof of the next theorem is similar to Theorem 1.3 and hence is omitted.

THEOREM 1.8. Every quasicomponent of an aposyndetic space is aposyndetic.

THEOREM 1.9. If $M$ is a Hausdorff aposyndetic continuum and $A$ is an A-set of $M$, then $A$ is an aposyndetic continuum.

Proof. The fact that $A$ is a continuum follows from [2, Theorem 15]. For each $p \in A$ and $q \in A-\{p\}$, since $M$ is aposyndetic, there exists a closed connected set $H$ such that $p \in H$ and $H \subset M-\{q\}$. By [2, Theorem 15], $H \cap A$ is closed and connected and $\operatorname{int}(H) \cap A$ is open in $A$ containing $p$ so the result follows.

DEFINITION 1.10. A simple chain is a finite collection $\left\{U_{1}, \ldots, U_{n}\right\}$ of point sets such that $U_{i} \cap U_{j} \neq \varnothing$ if and only if $|i-j| \leq 1$.

DEFINITION 1.11. For any two points $a$ and $b$ of a connected space $M$, let $E(a, b)=$ $\{x \in M: x$ separates $a$ from $b$ in $M\}$. The interval $a b$ of $M$, denoted by $I_{a b}$, is the set $E(a, b) \cup\{a, b\}$.

It is known that every interval in a connected semi-locally connected space is closed. The following theorem shows that the result holds in a connected aposyndetic space.

THEOREM 1.12. Every interval of a connected aposyndetic Hausdorff space is closed.

Proof. Let $I_{a b}$ be an interval of a connected aposyndetic Hausdorff space $M$. Assume $p$ is a limit point of $I_{a b}$ such that $p \notin I_{a b}$. Let $C$ be the component in $M-\{p\}$ containing $a$. Since $p \notin I_{a b}, b$ must be in $C$. That is, $\{a, b\} \subset C$. Suppose there exists an $x \in I_{a b}$ such that $x \notin C$. Let $M-\{x\}=A \cup B$, where $A$ and $B$ are separated with $a \in A$ and $b \in B$. Since $\{a, b\} \subset C \subseteq M-\{x\}=A \cup B, C \subseteq A$ or $C \subseteq B$. This implies $\{a, b\} \subset A$ or $\{a, b\} \subset B$, which yields a contradiction. Thus if $x \in I_{a b}$, then $x \in C$. For each $x \in C$, there exists a closed connected set $H_{x}$ such that $x \in \operatorname{int}\left(H_{x}\right)$ and $p \notin H_{x}$ so $\left\{\operatorname{int}\left(H_{x}\right): x \in C\right\}$ is an open cover of $C$. By [1, Theorem 3.4], there exists a simple chain $\left\{\operatorname{int}\left(H_{x_{1}}\right), \operatorname{int}\left(H_{x_{2}}\right), \ldots, \operatorname{int}\left(H_{x_{n}}\right)\right\}$ from $a$ to $b$. Suppose for some $x \in I_{a b}$, $x \notin \cup_{i=1}^{n} \operatorname{int}\left(H_{x_{i}}\right)$. Let $M-\{x\}=A \cup B$, where $A$ and $B$ are separated with $a \in A$ and $b \in B$. Since $\cup_{i=1}^{n} H_{x_{i}}$ is connected, $\cup_{i=1}^{n} H_{x_{i}} \subseteq A$ or $\cup_{i=1}^{n} H_{x_{i}} \subseteq B$. This implies $\{a, b\} \subset A$ or $\{a, b\} \subset B$, which is a contradiction. Hence $I_{a b} \subseteq \cup_{i=1}^{n} H_{x_{i}}$ and $p \notin \cup_{i=1}^{n} H_{x_{i}}$. The fact that $\cup_{i=1}^{n} H_{x_{i}}$ is closed implies $p$ is not a limit point of $I_{a b}$. This contradicts the original assumption, hence $I_{a b}$ is closed. 
THEOREM 1.13. Let $a, b$, and $p$ be three distinct points in $M$ such that $p \notin I_{a b}$. If $M$ is Hausdorff, connected, and aposyndetic at each point in $M-\{p\}$, then there exists a closed connected subset $N$ of $M$ with $\{a, b\} \subset N \subset M-\{p\}$.

Proof. Since $p \notin I_{a b}$, there exists a component $C$ of $M-\{p\}$ containing $\{a, b\}$. Since $M$ is aposyndetic at each point in $M-\{p\}$, for each $x \in C$, there exists a closed connected set $K_{x}$ such that $x \in \operatorname{int}\left(K_{x}\right) \subset K_{x} \subset M-\{p\}$ so $C \subset \cup_{x \in C} \operatorname{int}\left(K_{x}\right)$. By [1, Theorem 3.4], there is a simple chain $\left\{K_{x_{1}}, \ldots, K_{x_{n}}\right\}$ from $a$ to $b$ and $p \in \cup_{i=1}^{n} K_{x_{i}}$. The result follows by letting $N=\cup_{i=1}^{n} K_{x_{i}}$.

COROLlary 1.14. If $M$ is Hausdorff, connected, and aposyndetic at each point in $M-\{p\}$, where $p$ is a noncut point of $M$, then for each pair of points $a, b$ in $M-\{p\}$, there exists a closed connected subset $N$ of $M$ with $\{a, b\} \subset N \subset M-\{p\}$.

DefinITION 1.15. A subset $E$ of a space $M$ is an $E_{0}$-set of $M$ if and only if $E$ is nondegenerate, connected, has no cut point of itself, and is maximal with respect to these properties.

DEFINITION 1.16. Let $R$ be a relation on the nondegenerate connected space $M$ defined by $x R y$ if and only if no point of $M$ separates $x$ from $y$ in $M$. Thus $R$ is reflexive and symmetric on $M$. For each point $x$ of $M$ which is neither a cut point nor an end point $M$, then the set of all points $y$ of $M$ such that $x R y$ is called a simple link of $M$.

Definition 1.17. A space $M$ is paraseparable if and only if $M$ does not contain uncountably many disjoint open sets.

The next lemma is known and can be found in [4].

LEMMA 1.18. If $Y$ is an uncountable set of cut points of the paraseparable connected set $M$, then some two points of $Y$ are separated in $M$ by a third point of $Y$.

LEMMA 1.19. Every $E_{0}$-set of a paraseparable connected set $M$ contains at most countably many cut points of $M$.

Proof. Suppose that there exists an $E_{0}$-set $E$ of $M$ such that the set $Y$, which is the set of points in $E$ that are also cut points of $M$, is uncountable. By Lemma 1.18, some two points $a, b$ of $Y$ are separated in $M$ by a third point $x$ of $Y$. Let $M-\{x\}=A \cup B$, where $A$ and $B$ are separated with $a \in A$ and $b \in B$. Then $E \subset A \cup\{x\}$ or $E \subset B \cup\{x\}$, which implies $\{a, b\} \subset A$ or $\{a, b\} \subset B$. This contradicts the statement that $a, b$ are separated by $x$ in $M$.

THEOREM 1.20. Let $M$ be a connected, locally compact, paraseparable, and aposyndetic space. If $L$ is a nondegenerate subset of $M$, then $L$ is a simple link of $M$ if and only if $L$ is an $E_{0}$-set of $M$.

Proof. Let $L_{p}$ be a simple link containing $p$ in $M$. Suppose that $L_{p}$ has a cut point $x$. Then $L_{p}-\{x\}=A \cup B$, where $A$ and $B$ are separated in $L_{p}$ with $a \in A, b \in B$ for some $a, b$ in $L_{p}$. Since $x$ cannot separate $a$ and $b$ in $M$, by Theorem 1.13 , there exists a continuum $N$ such that $\{a, b\} \subset N \subset M-\{x\}$. Without loss of generality, assume that 
$N$ is irreducible between $a$ and $b$. Since $N \subset M-\{x\}$ and $L_{p}$ contains all irreducible continua containing $a$ and $b$ by [6, Chapter IV, Theorem 1.3], $N \subset L_{p}-\{x\}$ implies $N \subset A$ or $N \subset B$, which contradicts $\{a, b\} \subset N$. Therefore, $L_{p}$ has no cut point.

Suppose that there is a connected set $C$ such that $L_{p} \subset C$ and $C$ has no cut point. Let $x \in C-L_{p}$. Then there exists $y \in M$ such that $M-\{y\}=M_{x} \cup M_{p}$, where $M_{x}$ and $M_{p}$ are separated with $x \in M_{x}$ and $p \in M_{p}$. Since $C$ has no cut point of itself, $y \notin C$. Then $\{x, p\} \subset C \subset M-\{y\}$ implies $x \notin M_{x}$ or $p \notin M_{p}$, which leads to a contradiction. Therefore, $L_{p}$ is an $E_{0}$-set.

Conversely, let $E$ be an $E_{0}$-set. Since $E$ is closed and $M$ is locally compact, $E$ is locally compact and hence is uncountable because no locally compact connected set is the union of a nondegenerate countable collection of disjoint compact sets. Since $E$ is an $E_{0}$-set and $E$ contains at most countably many cut points of $M$, there exists $p \in E$ such that $p$ is neither a cut point nor an end point of $M$ by Lemma 1.19. Let $L_{p}$ be the simple link containing $p$. Suppose $E \nsubseteq L_{p}$. Let $q \in E-L_{p}$. Then there exists $x \in M$ such that $M-\{x\}=M_{p} \cup M_{q}$, where $M_{p}$ and $M_{q}$ are separated with $p \in M_{p}$ and $q \in M_{q}$. If $x \notin E$, then $E \subset M_{p}$ or $E \subset M_{q}$. This implies $\{p, q\} \subset M_{p}$ or $\{p, q\} \subset M_{q}$, which contradicts the fact that $p$ and $q$ are separated by $x$ in $M$. If $x \in E$, then $E-\{x\}=\left(E \cap M_{p}\right) \cup\left(E \cap M_{q}\right)$. This implies that $x$ is a cut point of $E$, which contradicts the fact that $E$ is an $E_{0}$-set. Therefore, $E \subseteq L_{p}$. Since it has been proved that every simple link is an $E_{0}$-set in a locally compact, paraseparable, aposyndetic space, $L_{p}=E$.

\section{Semi-locally connected spaces}

Definition 2.1. A space $M$ is semi-locally connected at a point $p$ of $M$ if and only if each open set containing $p$ contains an open set $V$ containing $p$ such that $M-V$ has at most a finite number of components. The space $M$ is semi-locally connected if and only if it is semi-locally connected at each point $p$ of $M$.

THEOREM 2.2. Every quasicomponent of a semi-locally connected space is semilocally connected.

Proof. Let $Q$ be a quasicomponent of a semi-locally connected space $M$ and $p \in Q$. Since $Q$ is an open subset of $M$ containing $p$ and $M$ is semi-locally connected, there is an open set $V$ in $M$ containing $p$ such that $p \in V$ and $M-V=\cup_{i=1}^{n} K_{i}$, where $K_{i}$ is a component of $M-V$. Now $Q-V=Q \cap(M-V)=Q \cap\left(\cup_{i=1}^{n} K_{i}\right)$, let $F=\left\{j: K_{j} \cap Q \neq\right.$ $\varnothing, j=1,2, \ldots, n\}$. Then for each $j \in F, K_{j} \subset Q$ because $Q$ is a component of $M$ by Theorem 1.2(a), and $K_{j}$ is a component of $Q-V$. Therefore, $Q-V=\cup_{j \in F} K_{i}$, which implies that $Q$ is semi-locally connected.

DEFINITION 2.3. A cyclic element of a connected space $M$ is a subset of $M$ which either consists of a single cut point or end point of $M$ or is an $E_{0}$-set of $M$.

THEOREM 2.4. (a) If $M$ is a paraseparable semi-locally connected continuum and $A$ is a closed set in $M$, then $A$ is an A-set in $M$ if and only if for each cyclic element $E$ of $M$ such that $A \cap E$ is nondegenerate, $E \subset A$.

(b) If $M$ is a paraseparable semi-locally connected continuum and $A$ is a nondegenerate subcontinuum in $M$, then $A$ is an $A$-set in $M$ if and only if $A$ is the union of cyclic elements of $M$. 
(c) If $M$ is a paraseparable semi-locally connected continuum, then every cyclic element of $M$ is an A-set of $M$.

Proof. (a) Let $A$ be an $A$-set of $M$ and let $E$ be a cyclic element of $M$ such that $A \cap E$ is nondegenerate. Let $p$ and $q$ be distinct points of $A \cap E$. Assume there exists a point $x$ in $E-A$. Let $K$ be the component of $M-A$ containing $x$ and let $\partial K=\{y\}$. Since $E$ is connected and it intersects both $K$ and $M-K, y \in E$. But then $y$ is a cut point of $E$. Now, either $p$ or $q$ is in $A \cap E-\{y\}$. Therefore, $E \subset A$.

Conversely, suppose that, for each cyclic element $E$ of $M$ such that $A \cap E$ is nondegenerate, $E \subset A$. Then the result follows from [6, Chapter IV, Theorem 3.3] and [2, Theorem 12].

(b) The result follows from [6, Chapter IV, Theorem 3.3] and [2, Theorem 12].

(c) Let $E$ be a cyclic element of $M$ and let $p \in E$. Then either $\{p\}=E$, or there is a cyclic element, namely $E$, of $M$ such that $p \in E$ and $E \subset E$. By part (a), $E$ is an $A$-set of $M$.

THEOREM 2.5. If $M$ is a paraseparable semi-locally connected continuum, then the intersection of a collection of A-sets of $M$ is itself an A-set of $M$.

Proof. Let $\varphi$ be a collection of $A$-sets of $M$, and let $A=\cap \mathscr{G}$. If $A \neq \varnothing$ and is degenerate, then $A$ is an $A$-set of $M$. Let $a \in A$, and for each $x$ in $A-\{a\}$, let $N_{x}$ be the irreducible subcontinuum of $M$ from $a$ to $x$. By [2, Theorem 18], for every $G \in \mathscr{G}$, $N_{x} \subset G$, so that $N_{x} \subset A$. Hence $A$ is a continuum. Suppose $p \in A$ and $\{p\} \neq A$. If $p$ is a cut point or an end point of $M$, then $\{p\}$ is a degenerate cyclic elements of $M$ and $\{p\} \subset A$. If $p$ is a non-cut point and a non-end point of $M$, then $p$ belongs to a nondegenerate cyclic element $E$ of $M$. By Theorem 2.4(a), $E \subset G$ for each $G \in \mathscr{G}$. Then $E \subset A$, and hence, again by Theorem 2.4(a), $A$ is an $A$-set.

DEFINITION 2.6. If $\varphi$ is a covering of a space $M$, then a subset $E$ of $M$ is said to be of diameter less than $\mathscr{G}$ if some element of $\mathscr{G}$ contains $E$. Let $\mathscr{F}$ be a family of subsets of a space $M$. Then $\mathscr{F}$ is called a null family if and only if for every open cover $\mathscr{G}$ of $M$, all but a finite number of members of $\mathscr{F}$ have diameter less than $\mathscr{G}$.

The following theorem established by Simone [5] is useful in proving the result: in a semi-locally connected space, the set of all components of the complement of an $A$-set is a null family.

THEOREM 2.7. Let $\varphi$ be a family of subsets of the compact space $M$. Then $\varphi$ is a null family if and only if for any two disjoint closed sets $A$ and $B$, at most a finite number of members of $\varphi$ intersect both $A$ and $B$.

THEOREM 2.8. If $M$ is a semi-locally connected continuum and $A$ is an A-set, then the set of all components of $M-A$ is a null family.

Proof. Let $H$ and $K$ be two disjoint closed subsets of $M$. Let $\left\{C_{i}: i \in I\right\}$ be the set of components of $M-A$ that intersect both $H$ and $K$. Assume $I$ is infinite. For each $i \in I$, let $\partial C_{i}=\left\{p_{i}\right\}$. If $\left\{p_{i}: i \in I\right\}$ is finite, then for some point $p \in A, p_{i}=p$ for infinitely many $C_{i}$. If $\left\{p_{i}: i \in I\right\}$ is infinite, then let $p$ be a limit point of $\left\{p_{i}: i \in I\right\}$. Since $A$ is closed, $p \in A$. One of the sets $H$ or $K$, say $K$, does not contain $p$. Let $U=M-K$. 
Then $K \subset M-U$ and $(M-U) \cap C_{i} \neq \varnothing$ for infinitely many $i \in I$. But this contradicts the fact that $M$ is semi-locally connected. Hence $I$ is finite. By Theorem 2.7, the set of all components of $M-A$ is a null family.

\section{Concerning nodal sets}

DEFINITION 3.1. A space $M$ is said to be strongly connected if and only if for each two points $a$ and $b$ in $M$, there exists a continuum $L$ in $M$ such that $L$ contains $a$ and $b$. A subset $N$ of a connected space $M$ is called a nodal set of $M$ if and only if $N$ is closed and $\partial N$ is degenerate.

Note that $N$ is an $A$-set and is connected.

DefinITION 3.2. A space $M$ is connected im kleinen at the point $p$ of $M$ if and only if each open set $U$ containing $p$ contains an open set $V$ containing $p$ such that each point of $V$ belongs to a connected set containing $p$ and lying in $U$.

THEOREM 3.3. If $M$ is a nondegenerate paraseparable connected space and $U$ is an open subset of $M$ such that $U$ is locally compact and each nondegenerate continuum in $U$ contains uncountably many cut points of $M$, then there exist two points $a$ and $b$ in $M$ that are separated in $M$ by uncountably many points of $M$.

Proof. Let $p \in U$. There exists an open set $V$ such that $p \in V, V \subset U$, and $\bar{V}$ is compact. Assume $M$ is not connected im kleinen at $p$. There exists a continuum of convergence $K$ in $\bar{V}$ such that $p \in K$. Then $K$ contains uncountably many cut points of $M$. By Lemma 1.18, some two points in $K$ are separated in $M$ by a third point of $K$. But this is impossible, since $K$ is a continuum of convergence in $M$. Hence $M$ is connected im kleinen at $p$. Then $U$ is connected im kleinen at $p$, and therefore, $U$ is locally connected.

Let $W$ be an open connected set such that $W \subset U$. Since $W$ is locally compact, $W$ is strongly connected. Let $a$ and $b$ be two points in $W, L$ be an irreducible continuum in $W$ from $a$ to $b$, and let $T$ be the set of all points of $L$ that separates $a$ from $b$ in $M$. Assume $T$ is countable. Let $S=(L-\{a, b\})-T$. Then $S$ contains uncountably many cut points of $M$. Hence, by Lemma 1.18, some two points of $S$ are separated in $M$ by a third point $x$ of $S$. By the irreducibility of $L, x$ separates $a$ from $b$ in $M$. But $x \notin T$, and this is a contradiction. Hence $T$ is uncountable.

THEOREM 3.4. If $M$ is a connected space, $a$ and $b$ are points of $M$, and $L$ is an irreducible continuum in $M$ from a to $b$, then $L-\{a, b\}$ contains no end point of $M$.

Proof. Let $L$ be an irreducible continuum in $M$ from $a$ to $b$. Assume $L$ contains an end point $p$ of $M$. Let $U$ be an open set containing $p$ such that $a$ and $b$ are not in $\bar{U}$. There exists an open set $V$ such that $p \in V, V \subset U$, and $\partial V$ is degenerate. Let $\partial V=\{q\}$. Then $L \cap U$ is separated from $L \cap(M-V)$, and so $q$ is a cut point of $L$. Now $L \cap[(M-V) \cup\{q\}]$ is a proper subcontinuum of $L$ from $a$ to $b$. This is a contradiction. Hence $L$ contains no end point of $M$.

THEOREM 3.5. If $M$ is a nondegenerate paraseparable connected space and $U$ is an open subset of $M$ such that $U$ is locally compact and $U$ contains at most a countable 
number of non-cut points of $M$ that are non-end points of $M$, then there exist two points $a$ and $b$ in $M$ that are separated in $M$ by uncountably many points of $M$.

Proof. Let $U$ be as described in the hypothesis. Let $K$ be a nondegenerate continuum in $U$. Then $K$ is uncountable, and $K$ contains at most a countable number of non-cut points of $M$ that are non-end points of $M$. Let $x$ and $y$ be distinct points of $K$, and let $L$ be an irreducible subcontinuum of $K$ from $x$ to $y$. By Theorem 3.4, $L-\{x, y\}$ contains no end point of $M$, and so $L$ contains uncountably many cut points of $M$. Then by Theorem 3.3, there exist two points $a$ and $b$ in $M$ that are separated in $M$ by uncountably many points of $M$.

The following lemma is known and can be found in [4].

LEMMA 3.6. If $a$ and $b$ are points of the paraseparable connected space $M$ and $H$ is an uncountable subset of $I_{a b}$, then there exists a countable subset $K$ of $H$ such that if $p$ is a point of $H-K$, then $M-\{p\}$ is the union of two separated connected sets one containing $a$ and the other containing $b$.

THEOREM 3.7. If $M$ is a nondegenerate paraseparable connected space and $U$ is an open subset of $M$ such that $U$ is locally compact and each nondegenerate continuum of $U$ contains uncountably many cut points of $M$, then there exist two disjoint nodal sets $H$ and $K$ of $M$ such that $M-H$ and $M-K$ are connected and $H$ and $K$ have nonempty interiors.

Proof. By Theorem 3.4 and Lemma 3.6, there exists an uncountable subset $T$ of $U$ such that each point of $T$ separates $M$ into two nonempty connected sets. Let $q \in T$, and let $M-\{q\}=A \cup B$, where $A$ and $B$ are two nonempty separated connected sets. Let $p \in T \cap A$. Then $M-\{p\}=E \cup F$, where $E$ and $F$ are two nonempty separated connected sets. Since $E \cup\{p\}$ is a closed connected subset of $A \cup B, E \cup\{p\} \subset A$. Also $B \cup\{q\}$ is a closed connected subset of $M$. It is easy to see that $E \cup\{p\}$ and $B \cup\{q\}$ are disjoint. Now let $H=E \cup\{p\}$ and $K=B \cup\{q\}$. Then $M-H=F$ and $M-K=A$, and $F$ and $A$ are both connected. Since $E$ and $B$ are open, $H$ and $K$ are closed. Hence $H$ and $K$ have nonempty interior and degenerate boundaries.

Lehman [3] stated the theorem: if $M$ is a connected space, $p$ is a cut point of $M$ and $M-\{p\}=H \cup K$, then $H \cup\{p\}$ and $K \cup\{p\}$ are nodal sets. Whyburn [6] established a similar theorem. These theorems follow immediately from the definition of nodal sets. The following theorem provides sufficient conditions for the existence of two nodal sets in nondegenerate locally compact paraseparable connected spaces; however, these nodal sets are disjoint and do not separate the space.

THEOREM 3.8. If $M$ is a nondegenerate locally compact paraseparable connected space, then there exist two disjoint nodal sets $H$ and $K$ of $M$ such that $M-H$ and $M-K$ are connected.

Proof. If each point of $M$ is a cut point of $M$, then the result follows from Theorem 3.7. If $p$ and $q$ are distinct non-cut points of $M$, then let $H=\{p\}$ and $K=\{q\}$. If $M$ has exactly one non-cut point $x$, then $U=M-\{x\}$. Now $U$ is locally compact, and each 
nondegenerate subcontinuum of $U$ contains uncountably many cut points of $M$. The results then follows from Theorem 3.7.

\section{REFERENCES}

[1] J. G. Hocking and G. S. Young, Topology, Addison-Wesley, Massachusetts, 1961.

[2] D. A. John, A-sets and abcohesive spaces, Missouri J. Math. Sci. 5 (1993), no. 2, 63-67.

[3] B. Lehman, Cyclic element theory in connected and locally connected Hausdorff spaces, Canad. J. Math. 28 (1976), no. 5, 1032-1050.

[4] R. L. Moore, Foundations of Point Set Theory, American Mathematical Society Colloquium Publications, vol. 13, American Mathematical Society, Rhode Island, 1962.

[5] J. N. Simone, Hereditarily locally connected continua and the Hahn-Mazurkiewicz problem, Ph.D. thesis, University of Missouri, Kansas City, 1976.

[6] G. T. Whyburn, Analytic Topology, American Mathematical Society Colloquium Publications, vol. 28, American Mathematical Society, Rhode Island, 1942.

DAVID A. John: Missouri WeSteRn STATE College, SAINT JosePH, MO 64507, USA

E-mail address: john@griffon.mwsc.edu

Shing S. So: Central Missouri State University, WARrensburg, MO 64093, USA

E-mail address: so@cmsu1.cmsu.edu 


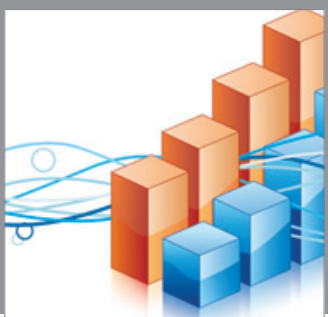

Advances in

Operations Research

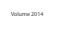

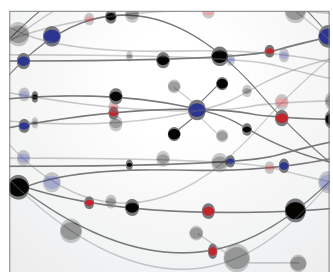

\section{The Scientific} World Journal
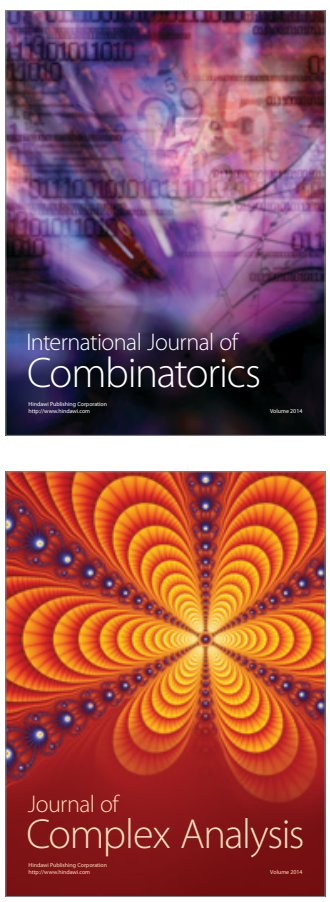

International Journal of

Mathematics and

Mathematical

Sciences
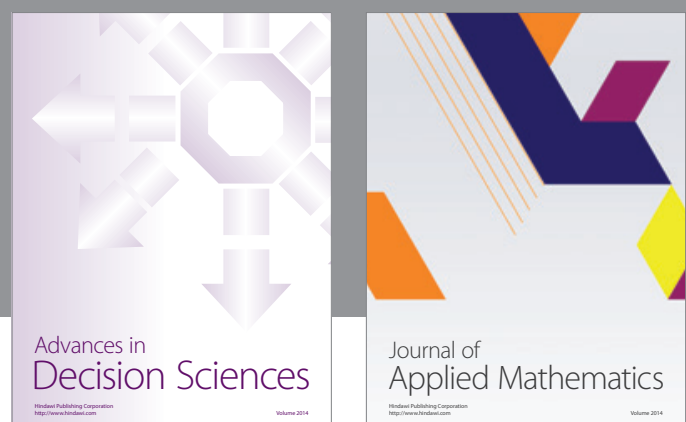

Journal of

Applied Mathematics
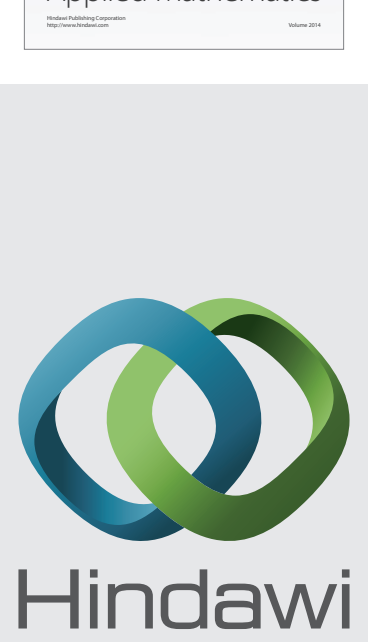

Submit your manuscripts at http://www.hindawi.com
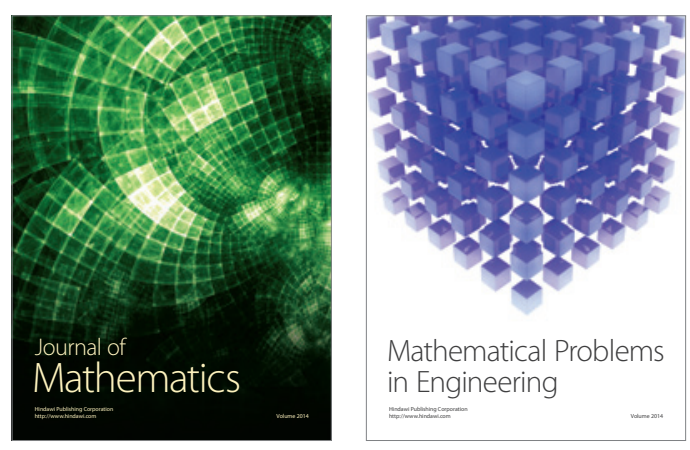

Mathematical Problems in Engineering
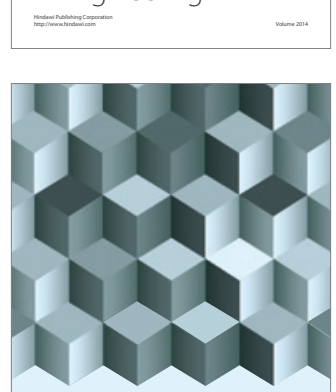

Journal of

Function Spaces
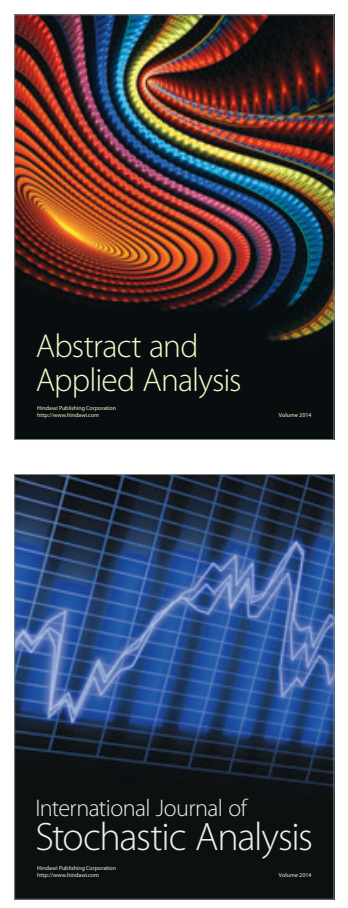

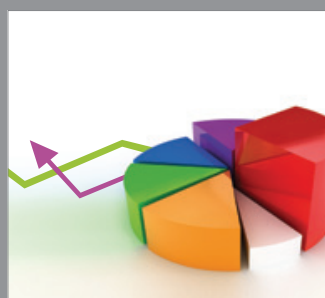

ournal of

Probability and Statistics

Promensencen
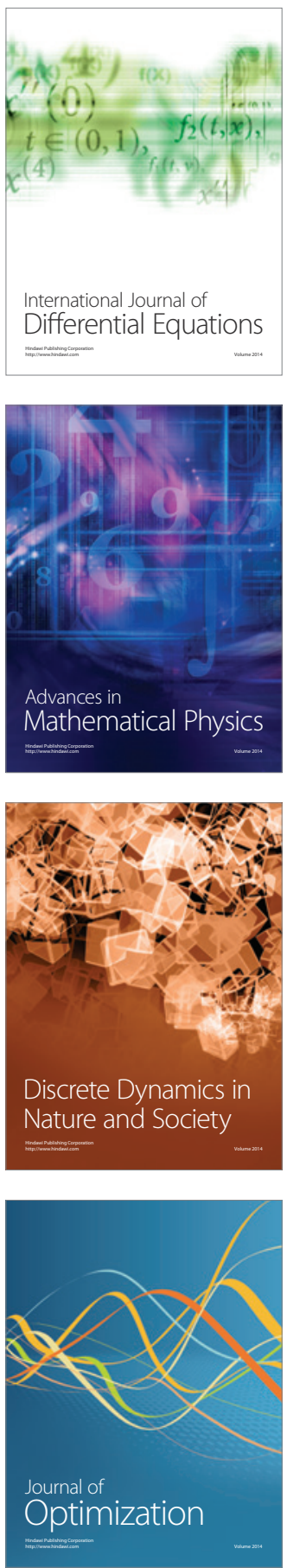